\title{
Fuzzy Controllers for Nonaffine-in-Control Singularly Perturbed Switched Systems
}

\author{
Linna Zhou, Qianjin Wang, Xiaoping Ma, and Chunyu Yang \\ School of Information and Electrical Engineering, China University of Mining and Technology, Xuzhou 221116, China \\ Correspondence should be addressed to Xiaoping Ma; xiaopingmacumt@126.com
}

Received 21 October 2014; Revised 14 December 2014; Accepted 14 December 2014

Academic Editor: Mohammed Chadli

Copyright (C) 2015 Linna Zhou et al. This is an open access article distributed under the Creative Commons Attribution License, which permits unrestricted use, distribution, and reproduction in any medium, provided the original work is properly cited.

\begin{abstract}
This paper investigates the problem of fuzzy controller design for nonaffine-in-control singularly perturbed switched systems (NCSPSSs). First, the NCSPSS is approximated by Takagi-Sugeno (T-S) models which include not only state but also control variables in the premise part of the rules. Then, a dynamic state feedback controller design method is proposed in terms of linear matrix inequalities. Under the controller, stability bound estimation problem of the closed-loop system is solved. Finally, an example is given to show the feasibility and effectiveness of the obtained methods.
\end{abstract}

\section{Introduction}

Switched systems, which consist of a finite number of subsystems and a logical rule governing the switching among the subsystems, are widely encountered in mechanical systems, power systems, and aircraft [1-3]. One of the basic problems of switched systems is the stability analysis of switched systems under arbitrary switching signals [1]. For this problem, it is necessary to assume that all the subsystems are asymptotically stable [4]. But this assumption is not sufficient for stability of the switched systems [4]. To assure stability under arbitrary switching signals, a minimal interval of time between two successive switchings (called dwell time) or a common Lyapunov function for all the subsystems is required [4-8].

On the other hand, many practical systems exhibit multiple time scale behavior, which can lead to high dimensionality and ill-conditioned numerical issues in the analysis and design problems $[9,10]$. Singular perturbation theory has been developed to deal with these problems [11, 12]. In this framework, the system analysis and synthesis problems are based on decomposing the system into fast and slow subsystems [9]. In the past several decades, the theory of singularly perturbed systems has attracted much attention and been applied to chemical processes [13], power systems [14], electromechanical systems [15], and so forth. On stability analysis and stabilization problems of SPSs, there exist two kinds of approaches. One is to present a condition for the existence of an upper bound $\varepsilon_{\max }$ for the singular perturbation parameter $\varepsilon$, such that the stability of the SPS is ensured for all $\varepsilon \in\left(0, \varepsilon_{\max }\right][16,17]$. The other is to propose a method to compute the stability bound $\varepsilon_{\max }[18,19]$.

Singularly perturbed switched systems (SPSSs) whose subsystems are SPSs are of practical interest in many industry processes $[20,21]$. An example is given in [22], where the tail end phase of the rolling process in a hot strip mill was modeled as a SPSS. The classical theory for SPSs is based on the Levinson-Tikhonov theorem [23] which shows, under the assumption that the SPS can be decomposed into slow and fast subsystems, that asymptotic stability of the subsystems is a sufficient condition for the stability of the SPS [16, 24]. However, this principle does not hold for SPSSs. It was shown that stability of the slow and fast switched subsystems is not sufficient for stability of the original SPSS and a dwell-time condition $[25,26]$ or a constraint taking into account the coupling between slow and fast subsystems has to be considered [20,21,27]. Although there have been plenty of methods to estimate the stability bound of SPSs, few approaches were proposed for SPSSs. The exception is given by [28], where a stability bound estimation method was proposed for linear SPSSs whose switching subsystems have the same fast subsystems. 
Fuzzy control has found a great variety of applications in control engineering $[29,30]$. Takagi-Sugeno (T-S) model based fuzzy control has become one of the most successful approaches since T-S model is a universal approximator for a wide class of nonlinear systems [31-34]. Recently, many researchers have focused on the analysis and design of T$S$ fuzzy SPSs. Stability analysis and stabilization problems for both continuous- and discrete-time T-S fuzzy SPSs were investigated in $[17,35]$, and some LMI-based approaches were derived. To get a satisfactory transient behavior, $H_{\infty}$ control for T-S fuzzy SPSs with pole placement constraints was considered in [36]. Using the results in $[17,35,36]$, the stability of the resulting closed-loop systems is only ensured for sufficiently small singular perturbation parameter $\varepsilon$. The problem of the stability bound estimation for T-S fuzzy systems was considered in $[37,38]$. Since the T-S models used in $[17,35-38]$ are only able to approximate affine nonlinear systems, the proposed methods can not be applied to nonaffine systems. However, there are many nonaffinein-control systems rising from practical applications, such as magnetic servo levitation control system [39], pendulum control systems [40], and chemical reactions [41].

Motivated by $[42,43]$, where a class of generalized T$S$ fuzzy dynamic models were proposed and shown to be universal approximators to general nonlinear systems, this paper investigates the problem of fuzzy controller design for nonaffine-in-control singularly perturbed switched systems (NCSPSSs). T-S models which include not only state but also control variables in the premise part of the rules are established to approximate the NCSPSS. A fuzzy dynamic state feedback controller is constructed. Under the assumption that the fast subsystems are stable, a controller design method is proposed. Then, the stability bound of the closed-loop system is addressed and an LMI-based method is established. Finally, a numerical example is included to illustrate the proposed results.

The rest of this paper is organized as follows. In Section 2, the problems under consideration are defined. The controller design method which is reduced to the feasibility of a set of $\varepsilon$-independent LMIs is given in Section 3. In Section 4, the estimation problem of stability bound is considered. An academic example is given in Section 5 to show the effectiveness and advantage of the obtained methods. Section 6 concludes the paper.

\section{System Description}

Consider the following nonlinear singularly perturbed switched system:

$$
\begin{aligned}
\dot{x}(t) & =f^{\sigma}(x(t), z(t), u(t)), \\
\varepsilon \dot{z}(t) & =g^{\sigma}(x(t), z(t), u(t)),
\end{aligned}
$$

where $x(t) \in \mathfrak{R}^{n}$ and $z(t) \in \mathfrak{R}^{m}$ are the states, $u(t) \in \mathfrak{R}^{p}$ is the input, and $\varepsilon>0$ denotes the singular perturbation parameter. $\sigma$ is the switching rule, which takes its values in the finite set $\Pi=\{1,2, \ldots, N\} . f$ and $g$ are the Lipschitz functions and nonaffine in control.
By using the algorithm in [42], each nonlinear subsystem $j(j=1, \ldots, N)$ could be represented by a T-S fuzzy model described by $r_{j}$ rules of the following form.

The $i$ th rule is

Plant Rule $i$ :

$$
\begin{aligned}
& \text { IF } x_{1}(t) \text { is } \mu_{i 1}^{j}, \ldots, x_{n}(t) \text { is } \mu_{i n}^{j} ; \\
& z_{1}(t) \text { is } v_{i 1}^{j}, \ldots, z_{m}(t) \text { is } v_{i n}^{j} ; \\
& u_{1}(t) \text { is } \xi_{i 1}^{j}, \ldots, u_{p}(t) \text { is } \xi_{i p}^{j} ; \\
& \text { THEN } \dot{x}(t)=A_{i 11}^{j} x(t)+A_{i 12}^{j} z(t)+B_{i 1}^{j} u(t) \\
& \qquad \dot{z}(t)=A_{i 21}^{j} x(t)+A_{i 22}^{j} z(t)+B_{i 2}^{j} u(t) \\
& \text { for } i=1,2, \ldots, r_{j},
\end{aligned}
$$

where $\mu_{i k}^{j}, v_{i k}^{j}, \xi_{i k}^{j}$ are fuzzy sets.

Denote

$$
\begin{array}{r}
h_{i}^{j}(x, z, u)=\frac{\prod_{k=1}^{n} \mu_{i k}^{j}\left(x_{k}\right) \prod_{k=1}^{m} v_{i k}^{j}\left(z_{k}\right) \prod_{k=1}^{p} \xi_{i k}^{j}\left(u_{k}\right)}{\sum_{i=1}^{r_{j}} \prod_{k=1}^{n} \mu_{i k}^{j}\left(x_{k}\right) \prod_{k=1}^{m} v_{i k}^{j}\left(z_{k}\right) \prod_{k=1}^{p} \xi_{i k}^{j}\left(u_{k}\right)}, \\
h_{i}^{j} \geq 0, \quad \sum_{i=1}^{r_{i}^{j}} h_{i}^{j}=1 .
\end{array}
$$

The scalars $h_{i}^{j}$ are the membership functions. By using the usual center-average defuzzifier, product inference, and singleton fuzzifier, the T-S fuzzy system can be inferred as

$$
\begin{aligned}
\dot{x}(t) & =\sum_{i=1}^{r^{j}} h_{i}^{j}\left[A_{i 11}^{j} x(t)+A_{i 12}^{j} z(t)+B_{i 1}^{j} u(t)+\Delta^{j}(x, z, u)\right], \\
\varepsilon \dot{z}(t) & =\sum_{i=1}^{r^{j}} h_{i}^{j}\left[A_{i 21}^{j} x(t)+A_{i 22}^{j} z(t)+B_{i 2}^{j} u(t)+\Delta^{j}(x, z, u)\right],
\end{aligned}
$$

where $\Delta^{j}(x, z, u)$ denotes the approximation error. According to [42], the error can be made arbitrarily small by choosing large enough number of fuzzy rules. Thus we omit the approximation error $\Delta^{j}(x, z, u)$ in the rest of the paper.

Remark 1 . The T-S models used in [17, 35-38] are only able to approximate affine nonlinear SPSs, while the T-S model in (4a) and (4b) can represent nonaffine-in-control systems given as in (la) and (lb), since the control variables are included in the premise part of the rules. As shown in the previous section, there are various examples of nonaffine-incontrol systems and SPSSs in practical applications. Therefore, the T-S fuzzy model in (4a) and (4b) will lay a foundation for fuzzy control of nonaffine-in-control SPSSs and can be applied to various applications such as aircraft control, electrical and electromechanical systems, and chemical reactions. 
The design of state feedback stabilizing fuzzy controllers for the fuzzy system (4a) and (4b) is based on the dynamic state feedback control method. The fuzzy controller is described by the following.

The $i$ th rule is

Plant Rule $i$ :

$$
\begin{aligned}
& \text { IF } x_{1}(t) \text { is } \mu_{i 1}^{j}, \ldots, x_{n}(t) \text { is } \mu_{i n}^{j} ; \\
& z_{1}(t) \text { is } v_{i 1}^{j}, \ldots, z_{m}(t) \text { is } v_{i n}^{j} ; \\
& u_{1}(t) \text { is } \xi_{i 1}^{j}, \ldots, u_{p}(t) \text { is } \xi_{i p}^{j} ; \\
& \text { THEN } \dot{u}(t)=D_{i}^{j} x(t)+M_{i}^{j} z(t)+N_{i}^{j} u(t) \\
& \quad \text { for } i=1,2, \ldots, r_{j} .
\end{aligned}
$$

Because the controller rules are the same as the plant rules, the controller is given as follows:

$$
\dot{u}(t)=\sum_{i=1}^{r^{j}} h_{i}^{j}\left[D_{i}^{j} x(t)+M_{i}^{j} z(t)+N_{i}^{j} u(t)\right] .
$$

Then we have the closed-loop system:

$$
\begin{aligned}
\dot{u}(t) & =\sum_{i=1}^{r^{j}} h_{i}^{j}\left[D_{i}^{j} x(t)+M_{i}^{j} z(t)+N_{i}^{j} u(t)\right], \\
\dot{x}(t) & =\sum_{i=1}^{r^{j}} h_{i}^{j}\left[A_{i 11}^{j} x(t)+A_{i 12}^{j} z(t)+B_{i 1}^{j} u(t)\right], \\
\varepsilon \dot{z}(t) & =\sum_{i=1}^{r^{j}} h_{i}^{j}\left[A_{i 21}^{j} x(t)+A_{i 22}^{j} z(t)+B_{i 2}^{j} u(t)\right],
\end{aligned}
$$

which can be rewritten in the following compact form:

$$
\begin{gathered}
\dot{\eta}(t)=\sum_{i=1}^{r^{j}} h_{i}^{j}\left[\bar{A}_{i 11}^{j} \eta(t)+\bar{A}_{i 12}^{j} z(t)+\bar{B}_{i 1}^{j} v(t)\right], \\
\varepsilon \dot{z}(t)=\sum_{i=1}^{r^{j}} h_{i}^{j}\left[\bar{A}_{i 21}^{j} \eta(t)+\bar{A}_{i 22}^{j} z(t)\right],
\end{gathered}
$$

where

$$
\begin{gathered}
\eta(t)=\left[\begin{array}{l}
u(t) \\
x(t)
\end{array}\right], \quad \bar{A}_{i 11}^{j}=\left[\begin{array}{cc}
0 & 0 \\
B_{i 1}^{j} & A_{i 11}^{j}
\end{array}\right], \\
\bar{A}_{i 12}^{j}=\left[\begin{array}{c}
0 \\
A_{i 12}^{j}
\end{array}\right], \quad \bar{B}_{i 1}^{j}=\left[\begin{array}{l}
I \\
0
\end{array}\right], \\
\bar{A}_{i 21}^{j}=\left[\begin{array}{ll}
B_{i 2}^{j} & A_{i 21}^{j}
\end{array}\right], \quad \bar{A}_{i 22}^{j}=A_{i 22}^{j}, \\
K_{i}^{j}=\left[\begin{array}{ll}
N_{i}^{j} & D_{i}^{j}
\end{array}\right], \quad v(t)=K_{i}^{j} \eta(t)+M_{i}^{j} z(t) .
\end{gathered}
$$

Upon introducing the indicator function

$$
\theta(t)=\left[\theta_{1}(t), \ldots, \theta_{N}(t)\right],
$$

where $\theta_{j}(t)=1$ if the switching system is in mode $j$ and $\theta_{j}(t)=0$ if it is in a different mode, one can write the whole closed-loop system corresponding to (1a) and (1b) as follows:

$$
\begin{gathered}
\dot{\eta}(t)=\sum_{j=1}^{N} \sum_{i=1}^{r^{j}} \theta_{j} h_{i}^{j}\left[\bar{A}_{i 11}^{j} \eta(t)+\bar{A}_{i 12}^{j} z(t)+\bar{B}_{i 1}^{j} v(t)\right], \\
\varepsilon \dot{z}(t)=\sum_{j=1}^{N} \sum_{i=1}^{r^{j}} \theta_{j} h_{i}^{j}\left[\bar{A}_{i 21}^{j} \eta(t)+\bar{A}_{i 22}^{j} z(t)\right] .
\end{gathered}
$$

Assume that $\bar{A}_{i 22}^{j}, j=1, \ldots, N, i=1, \ldots, r^{j}$, are Hurwitz matrices, which is a common assumption for investigations on standard SPSs. Then the corresponding slow and fast subsystems of the SPF (11a) and (11b) are obtained as follows:

$$
\begin{gathered}
\dot{\eta}_{s}(t)=\sum_{j=1}^{N} \sum_{i=1}^{r^{j}} \theta_{j} h_{i}^{j}\left[\bar{A}_{i s}^{j} \eta_{s}(t)+\bar{B}_{i s}^{j} v_{s}(t)\right], \\
\varepsilon \dot{z}_{f}(t)=\sum_{j=1}^{N} \sum_{i=1}^{r^{j}} \theta_{j} h_{i}^{j}\left[\bar{A}_{i 22}^{j} z_{f}(t)\right],
\end{gathered}
$$

where $\bar{A}_{i s}^{j}=\bar{A}_{i 11}^{j}-\bar{A}_{i 12}^{j} \bar{A}_{i 22}^{j-1} \bar{A}_{i 21}^{j}, \bar{B}_{i s}^{j}=\bar{B}_{i 1}^{j}$.

\section{Controller Design}

This section will present a controller design method.

Theorem 2. If there exist matrices $P_{s}=\left(P_{s}\right)^{T}>0, P_{f}=\left(P_{f}\right)^{T}>$ $0, Q_{i s}^{j}=\left(Q_{i s}^{j}\right)^{T}>0, Q_{i f}^{j}=\left(Q_{i f}^{j}\right)^{T}>0, Z_{i s}^{j}$, and $Z_{i f}^{j}$ of appropriate dimensions satisfying the LMIs,

$$
\begin{gathered}
\bar{A}_{i s}^{j} P_{s}+P_{s}\left(\bar{A}_{i s}^{j}\right)^{T}+\bar{B}_{i s}^{j} Z_{i s}^{j}+\left(\bar{B}_{i s}^{j} Z_{i s}^{j}\right)^{T}+Q_{i s}^{j}<0, \\
\bar{A}_{i 22}^{j} P_{f}+P_{f}\left(\bar{A}_{i 22}^{j}\right)^{T}+Q_{i f}^{j}<0, \\
{\left[\begin{array}{cccc}
G_{i}^{j} & \star & \star & \star \\
F_{i}^{j} & Q_{i f}^{j} & \star & \star \\
Y_{i}^{j} & 0 & P_{f} & \star \\
\left(\bar{B}_{i 1}^{j} Z_{i f}^{j}\right)^{T}+Y_{i}^{j} & 0 & 0 & P_{f}
\end{array}\right]>0,}
\end{gathered}
$$

where $j=1, \ldots, N, i=1, \ldots, r^{j}, Y_{i}^{j}=-\sum_{k=1}^{N} \sum_{l=1}^{r_{k}}\left(\bar{A}_{l 22}^{k}\right)^{-1} \times$ $\left.\bar{A}_{l 21}^{k} P_{s}\right|_{(k, l) \neq(j, i)}, G_{i}^{j}=Q_{i s}^{j}-\bar{A}_{i 12}^{j} Y_{i}^{j}-\left(\bar{A}_{i 12}^{j} Y_{i}^{j}\right)^{T}$, and $F_{i}^{j}=$ $-\left(\bar{A}_{i 22}^{j} Y_{i}^{j}+P_{f}\left(\bar{A}_{i 12}^{j}\right)^{T}+\left(Z_{i f}^{j}\right)^{T}\left(\bar{B}_{i 1}^{j}\right)^{T}\right)$, then there exists a positive scalar $\varepsilon_{\max }$ such that, for all $\varepsilon \in\left(0, \varepsilon_{\max }\right]$, the closed-loop system (11a) and (11b) is stable and the controller gains are

$$
L_{i}^{j} \triangleq\left[\begin{array}{lll}
N_{i}^{j} & D_{i}^{j} & M_{i}^{j}
\end{array}\right]=\left[K_{i s}^{j}+K_{i f}^{j}\left(\bar{A}_{i 22}^{j}\right)^{-1} \bar{A}_{i 21}^{j} K_{i f}^{j}\right]
$$

with $K_{i s}^{j}=Z_{i s}^{j} P_{s}^{-1}, K_{i f}^{j}=Z_{i f}^{j} P_{f}^{-1}$. 
Proof. Let

$$
\begin{aligned}
P_{1}(\varepsilon) & =\varepsilon P_{s}, \\
P_{2}(\varepsilon) & =\varepsilon P_{2}=-\varepsilon \sum_{k=1 l=1}^{N} \sum_{l=1}^{r_{k}}\left(\bar{A}_{l 22}^{k}\right)^{-1} \bar{A}_{l 21}^{k} P_{s}, \\
P_{3}(\varepsilon) & =P_{f}+\varepsilon P_{2} P_{s}^{-1} P_{2}^{T}, \\
Z_{i 1}^{j}(\varepsilon) & =\varepsilon\left(Z_{i s}^{j}+Z_{i f}^{j} P_{f}^{-1} Y_{i}^{j}\right), \\
Z_{i 2}^{j}(\varepsilon) & =Z_{i f}^{j}+\varepsilon Z_{i s}^{j} P_{s}^{-1} P_{2}^{T}, \\
Q_{i 1}^{j}(\varepsilon) & =\varepsilon\left(Q_{i s}^{j}-\left(\bar{A}_{i 12}^{j}+\bar{B}_{i 1}^{j} Z_{i f}^{j} P_{f}^{-1}\right) Y_{i}^{j}\right. \\
Q_{i 2}^{j}(\varepsilon) & \left.\left.=-\left(\bar{A}_{i 12}^{j}+\bar{B}_{i 1}^{j} Z_{i f}^{j} P_{f}^{-1}\right) Y_{i}^{j}\right]^{T}\right), \\
Q_{i 3}^{j}(\varepsilon) & =\varepsilon^{-1} Q_{i f}^{j} .
\end{aligned}
$$

It follows from (15) that

$$
\left[\begin{array}{cccc}
G_{i}^{j}+\bar{B}_{i 1}^{j} Z_{i f}^{j} P_{f}^{-1}\left(\bar{B}_{i 1}^{j} Z_{i f}^{j}\right)^{T}+2\left(Y_{i}^{j}\right)^{T} P_{f}^{-1} Y_{i}^{j} & \star & \star & \star \\
F_{i}^{j} & Q_{i f}^{j} & \star & \star \\
Y_{i}^{j} & 0 & P_{f} & \star \\
\left(\bar{B}_{i 1}^{j} Z_{i f}^{j}\right)^{T}+Y_{i}^{j} & 0 & 0 & P_{f}
\end{array}\right]
$$

$>0$.

Using the Schur complement, inequality (18) implies that

$$
\left[\begin{array}{cc}
Q_{i s}^{j}-\left(\bar{A}_{i 12}^{j}+\bar{B}_{i 1}^{j} Z_{i f}^{j} P_{f}^{-1}\right) Y_{i}^{j}-\left[\left(\bar{A}_{i 12}^{j}+\bar{B}_{i 1}^{j} Z_{i f}^{j} P_{f}^{-1}\right) Y_{i}^{j}\right]^{T} & \star \\
F_{i}^{j} & Q_{i f}^{j}
\end{array}\right]
$$

$>0$

which is equivalent to

$$
\left[\begin{array}{ccc}
\varepsilon\left(Q_{i s}^{j}-\left(\bar{A}_{i 12}^{j}+\bar{B}_{i 1}^{j} Z_{i f}^{j} P_{f}^{-1}\right) Y_{i}^{j}-\left[\left(\bar{A}_{i 12}^{j}+\bar{B}_{i 1}^{j} Z_{i f}^{j} P_{f}^{-1}\right) Y_{i}^{j}\right]^{T}\right) & \star \\
F_{i}^{j} & \varepsilon^{-1} Q_{i f}^{j}
\end{array}\right]
$$$$
>0, \quad \forall \varepsilon>0 .
$$

Then we have

$$
\left[\begin{array}{cc}
Q_{i 1}^{j}(\varepsilon) & \star \\
Q_{i 2}^{j}(\varepsilon) & Q_{i 3}^{j}(\varepsilon)
\end{array}\right]>0 .
$$

System (8a) and (8b) can be rewritten as

$$
\dot{\chi}(t)=\sum_{j=1}^{N} \sum_{i=1}^{r^{j}} \theta_{j} h_{i}^{j}\left[\left(\bar{A}_{i}^{j}(\varepsilon)+\bar{B}_{i}^{j} L_{i}^{j}\right) \chi(t)\right],
$$

where

$$
\begin{gathered}
\chi=\left[\begin{array}{c}
\eta \\
z
\end{array}\right], \quad \bar{A}_{i}^{j}(\varepsilon)=\left[\begin{array}{cc}
\bar{A}_{i 11}^{j} & \bar{A}_{i 12}^{j} \\
\varepsilon^{-1} \bar{A}_{i 21}^{j} & \varepsilon^{-1} \bar{A}_{i 22}^{j}
\end{array}\right], \\
\bar{B}_{i}^{j}=\left[\begin{array}{c}
\bar{B}_{i 1}^{j} \\
0
\end{array}\right], \quad L_{i}^{j}=\left[\begin{array}{ll}
K_{i}^{j} & M_{i}^{j}
\end{array}\right] .
\end{gathered}
$$

Let

$$
P(\varepsilon)=\left[\begin{array}{cc}
P_{1}(\varepsilon) & \star \\
P_{2}(\varepsilon) & P_{3}(\varepsilon)
\end{array}\right] .
$$

Define $X_{i 1}^{j}(\varepsilon), X_{i 2}^{j}(\varepsilon)$, and $X_{i 3}^{j}(\varepsilon)$ as

$$
\begin{aligned}
X_{i 1}^{j}(\varepsilon)= & \bar{A}_{i 11}^{j} P_{1}(\varepsilon)+\left(\bar{A}_{i 11}^{j} P_{1}(\varepsilon)\right)^{T}+\bar{A}_{i 12}^{j} P_{2}(\varepsilon) \\
& +\left(\bar{A}_{i 12}^{j} P_{2}(\varepsilon)\right)^{T}+\bar{B}_{i 1}^{j} Z_{i 1}^{j}(\varepsilon) \\
& +\left(\bar{B}_{i 1}^{j} Z_{i 1}^{j}(\varepsilon)\right)^{T}+Q_{i 1}^{j}(\varepsilon), \\
X_{i 2}^{j}(\varepsilon)= & \varepsilon^{-1} \bar{A}_{i 22}^{j} P_{2}(\varepsilon)+\varepsilon^{-1} \bar{A}_{i 21}^{j} P_{1}(\varepsilon)+P_{3}(\varepsilon)\left(\bar{A}_{i 12}^{j}\right)^{T} \\
& +\left(\bar{A}_{i 11}^{j} P_{2}^{T}(\varepsilon)\right)^{T}+\left(\bar{B}_{i 1}^{j} Z_{i 2}^{j}(\varepsilon)\right)^{T}+Q_{i 2}^{j}(\varepsilon), \\
X_{i 3}^{j}(\varepsilon)= & \varepsilon^{-1}\left(\bar{A}_{i 22}^{j} P_{3}(\varepsilon)+\left(\bar{A}_{i 22}^{j} P_{3}(\varepsilon)\right)^{T}\right. \\
& \left.\quad+\bar{A}_{i 21}^{j} P_{2}^{T}(\varepsilon)+\left(\bar{A}_{i 21}^{j} P_{2}^{T}(\varepsilon)\right)^{T}+Q_{i 3}^{j}(\varepsilon)\right) .
\end{aligned}
$$

By simple calculation, we have

$$
\begin{gathered}
X_{i 1}^{j}(\varepsilon)=\varepsilon\left(\bar{A}_{i s}^{j} P_{s}+P_{s}\left(\bar{A}_{i s}^{j}\right)^{T}+\bar{B}_{i s}^{j} Z_{i s}^{j}\right. \\
\left.+\left(\bar{B}_{i s}^{j} Z_{i s}^{j}\right)^{T}+Q_{i s}^{j}\right)=\varepsilon X_{i s}^{j}, \\
X_{i 2}^{j}(\varepsilon)=\varepsilon\left(P_{2} P_{s}^{-1} P_{2}^{T}\left(\bar{A}_{i 12}^{j}\right)^{T}+\left(\bar{A}_{i 11}^{j} P_{2}^{T}\right)^{T}\right. \\
\left.+\left(\bar{B}_{i 1}^{j} Z_{i s}^{j} P_{s}^{-1} P_{2}^{T}\right)^{T}\right)=\varepsilon X_{i 2}^{j}, \\
X_{i 3}^{j}(\varepsilon)=\varepsilon^{-1}\left(\bar{A}_{i 22}^{j} P_{f}+P_{f}\left(\bar{A}_{i 22}^{j}\right)^{T}+Q_{i f}^{j}+O(\varepsilon)\right) \\
=\varepsilon^{-1}\left(X_{i f}^{j}+O(\varepsilon)\right) .
\end{gathered}
$$

LMI conditions (13) and (14) imply that

$$
X_{i s}^{j}<0, \quad X_{i f}^{j}<0
$$

which indicates that there exists a positive scalar $\varepsilon_{0}>0$, such that, for any $\varepsilon \in\left(0, \varepsilon_{0}\right]$, it holds that

$$
\left[\begin{array}{cc}
X_{i 1}^{j}(\varepsilon) & \star \\
X_{i 2}^{j}(\varepsilon) & X_{i 3}^{j}(\varepsilon)
\end{array}\right]=\left[\begin{array}{cc}
\varepsilon X_{i s}^{j} & \star \\
\varepsilon X_{i 2}^{j} & \varepsilon^{-1}\left(X_{i f}^{j}+O(\varepsilon)\right)
\end{array}\right]<0 .
$$


On the other hand, it is easy to see that

$$
\begin{aligned}
{\left[\begin{array}{cc}
X_{i 1}^{j}(\varepsilon) & \star \\
X_{i 2}^{j}(\varepsilon) & X_{i 3}^{j}(\varepsilon)
\end{array}\right]=} & \bar{A}_{i}^{j}(\varepsilon) P(\varepsilon)+\bar{B}_{i}^{j} Z_{i}^{j}(\varepsilon) \\
& +Q_{i}^{j}(\varepsilon)+P(\varepsilon)\left(\bar{A}_{i}^{j}(\varepsilon)\right)^{T} \\
& +\left(\bar{B}_{i}^{j} Z_{i}^{j}(\varepsilon)\right)^{T} .
\end{aligned}
$$

Then it follows from (28) and (29) that

$$
\begin{array}{r}
\bar{A}_{i}^{j}(\varepsilon) P(\varepsilon)+\bar{B}_{i}^{j} Z_{i}^{j}(\varepsilon)+P(\varepsilon)\left(\bar{A}_{i}^{j}(\varepsilon)\right)^{T}+\left(\bar{B}_{i}^{j} Z_{i}^{j}(\varepsilon)\right)^{T}<0 \\
\varepsilon \in\left(0, \varepsilon_{0}\right] .
\end{array}
$$

$$
\begin{aligned}
& \text { Let } L_{i}^{j}(\varepsilon)=Z_{i}^{j}(\varepsilon)(P(\varepsilon))^{-1} \text {; then } \\
& L_{i}^{j}(\varepsilon)=Z_{i}^{j}(\varepsilon)(P(\varepsilon))^{-1} \\
& =\left[\varepsilon\left(Z_{i s}^{j}+Z_{i f}^{j} P_{f}^{-1} Y_{i}^{j}\right) Z_{i f}^{j}+\varepsilon Z_{i s}^{j} P_{s}^{-1} P_{2}^{T}\right] \\
& \times\left[\begin{array}{cc}
\varepsilon P_{s} & \varepsilon P_{2}^{T} \\
\varepsilon P_{2} & P_{f}+\varepsilon P_{2} P_{s}^{-1} P_{2}^{T}
\end{array}\right]^{-1} \\
& =\left[Z_{i s}^{j}+Z_{i f}^{j} P_{f}^{-1} Y_{i}^{j} Z_{i f}^{j}+\varepsilon Z_{i s}^{j} P_{s}^{-1} P_{2}^{T}\right] \\
& \times\left[\begin{array}{cc}
P_{s} & \varepsilon P_{2}^{T} \\
P_{2} & P_{f}+\varepsilon P_{2} P_{s}^{-1} P_{2}^{T}
\end{array}\right]^{-1} \\
& \longrightarrow\left[Z_{i s}^{j}+Z_{i f}^{j} P_{f}^{-1} Y_{i}^{j} Z_{i f}^{j}\right]\left[\begin{array}{cc}
P_{s} & 0 \\
P_{2} & P_{f}
\end{array}\right]^{-1} \quad(\text { as } \varepsilon \longrightarrow 0) \\
& =\left[K_{i s}^{j}+K_{i f}^{j}\left(A_{i 22}^{j}\right)^{-1} A_{i 21}^{j} K_{i f}^{j}\right],
\end{aligned}
$$

where $K_{i s}^{j}=Z_{i s}^{j} P_{s}^{-1}$ and $K_{i f}^{j}=Z_{i f}^{j} P_{f}^{-1}$.

Thus, for sufficiently small $\varepsilon$, the controller gains can be chosen as

$$
L_{i}^{j}=\left[K_{i s}^{j}+K_{i f}^{j}\left(A_{i 22}^{j}\right)^{-1} A_{i 21}^{j} K_{i f}^{j}\right] .
$$

Then, by (30), (31), and (32), there exists a positive scalar $\varepsilon_{\max }<\varepsilon_{0}$, such that

$$
\begin{array}{r}
\left(\bar{A}_{i}^{j}(\varepsilon)+\bar{B}_{i}^{j} L_{i}^{j}\right) P(\varepsilon)+P(\varepsilon)\left(\bar{A}_{i}^{j}(\varepsilon)+\bar{B}_{i}^{j} L_{i}^{j}\right)^{T}<0, \\
\forall \varepsilon \in\left(0, \varepsilon_{\max }\right]
\end{array}
$$

which is equivalent to

$$
\begin{aligned}
\prod_{i}^{j}(\varepsilon) \triangleq & P^{-1}(\varepsilon)\left[\left(\bar{A}_{i}^{j}(\varepsilon)+\bar{B}_{i}^{j} L_{i}^{j}\right)\right] \\
& +\left[\left(\bar{A}_{i}^{j}(\varepsilon)+\bar{B}_{i}^{j} L_{i}^{j}\right)\right]^{T} P^{-1}(\varepsilon)<0, \quad \forall \varepsilon \in\left(0, \varepsilon_{\max }\right] .
\end{aligned}
$$

Define Lyapunov function

$$
V(\chi(t))=\chi^{T}(t) P^{-1}(\varepsilon) \chi(t)
$$

Computing the derivative of $V(\chi(t))$ along the trajectories of system (22) and taking into account (34), we have

$$
\begin{aligned}
\dot{V}( & (t)) \\
& =2 \chi^{T}(t) P^{-1}(\varepsilon) \dot{\chi}(t) \\
& =2 \chi^{T}(t) P^{-1}(\varepsilon)\left(\sum_{j=1}^{N} \sum_{i=1}^{r^{j}} \theta_{j} h_{i}^{j}\left[\left(\bar{A}_{i}^{j}(\varepsilon)+\bar{B}_{i}^{j} L_{i}^{j}\right) \chi(t)\right]\right) \\
& =2 \sum_{j=1}^{N} \sum_{i=1}^{r^{j}} \theta_{j} h_{i}^{j} \chi^{T}(t) P^{-1}(\varepsilon)\left[\left(\bar{A}_{i}^{j}(\varepsilon)+\bar{B}_{i}^{j} L_{i}^{j}\right) \chi(t)\right] \\
& =\sum_{j=1}^{N} \sum_{i=1}^{r^{j}} \theta_{j} h_{i}^{j} \chi^{T}(t) \prod_{i}^{j}(\varepsilon) \chi(t)<0, \\
& \forall \varepsilon \in\left(0, \varepsilon_{\max }\right], \quad \chi(t) \neq 0 .
\end{aligned}
$$

Therefore, the closed-loop system (11a) and (11b) is stable for all $\varepsilon \in\left(0, \varepsilon_{\max }\right]$. This completes the proof.

Remark 3. LMIs (13), (14), and (15) are independent of the singular perturbation parameter $\varepsilon$ and thus well-defined. The feasibility of the LMIs can be checked by the existing algorithms [44]. If the LMIs are feasible, an $\varepsilon$-independent stabilization controller can be obtained when $\varepsilon$ is small enough.

Remark 4. In [17, 35-38], some T-S model based control approaches have been proposed for nonlinear SPSs. The results are based on the parallel distributed compensation (PDC) scheme and can design state feedback controllers for affine-in-control nonlinear systems. In contrast, this paper employed a dynamic state feedback fuzzy controller and can be applied to nonaffine-in-control singularly perturbed switched systems.

Remark 5. LMIs (14) imply that the controller design method is valid only if the fast subsystem is open-loop stable, which limits the method to standard NCSPSS. Control of nonstandard NCSPSS is still an open problem.

Remark 6. T-S models, which use a set of fuzzy rules to describe a nonlinear system in terms of a set of local linear models, offer an efficient approach to stability analysis and controller design of complex nonlinear systems. In this framework, most of the stability analysis and controller design problems can be reduced to solve LMI problems. A larger number of individual subsystems or fuzzy rules will lead to larger computational burden. Fortunately, there have been some efficient algorithms to deal with LMI problems with reasonable large dimensions [44]. 


\section{Stability Bound Analysis}

Stability bound is a key stability index of SPSs. Theorem 2 guarantees the existence of the stability bound $\varepsilon_{\max }$. This section will propose a method to estimate the stability bound of the closed-loop system. To begin with, the closed-loop system is written as

$$
\begin{gathered}
\dot{\eta}(t)=\sum_{i=1}^{r^{j}} h_{i}^{j}\left[\widetilde{A}_{i 11}^{j} \eta(t)+\widetilde{A}_{i 12}^{j} z(t)\right], \\
\varepsilon \dot{z}(t)=\sum_{i=1}^{r^{j}} h_{i}^{j}\left[\widetilde{A}_{i 21}^{j} \eta(t)+\widetilde{A}_{i 22}^{j} z(t)\right],
\end{gathered}
$$

where

$$
\widetilde{A}_{i 11}^{j}=\left[\begin{array}{cc}
N_{i}^{j} & D_{i}^{j} \\
B_{i 1}^{j} & A_{i 11}^{j}
\end{array}\right], \quad \widetilde{A}_{i 12}^{j}=\left[\begin{array}{c}
M_{i}^{j} \\
A_{i 12}^{j}
\end{array}\right] .
$$

Theorem 7. If there exists matrices $P_{s}=\left(P_{s}\right)^{T}>0, P_{f}=$ $\left(P_{f}\right)^{T}>0, Q_{i s}^{j}=\left(Q_{i s}^{j}\right)^{T}>0$, and $Q_{i f}^{j}=\left(Q_{i f}^{j}\right)^{T}>0$ of appropriate dimensions, such LMIs,

$$
\begin{gathered}
X_{i s}^{j}<0, \\
X_{i f}^{j}<0 \\
{\left[\begin{array}{cc}
X_{i s}^{j} & \star \\
\varepsilon_{\max } X_{i 2}^{j} & X_{i f}^{j}+\varepsilon_{\max } M
\end{array}\right]<0,} \\
{\left[\begin{array}{c}
Q_{i s}^{j}-\widetilde{A}_{i 12}^{j} Y_{i}^{j}-\left(\widetilde{A}_{i 12}^{j} Y_{i}^{j}\right)^{T} \star \\
-\left(\widetilde{A}_{i 22}^{j} Y_{i}^{j}+P_{f}\left(\widetilde{A}_{i 12}^{j}\right)^{T}\right) Q_{i f}^{j}
\end{array}\right]>0,}
\end{gathered}
$$

are verified for $j=1, \ldots, N, i=1, \ldots, r^{j}$ with

$$
\begin{gathered}
X_{i s}^{j}=\widetilde{A}_{i s}^{j} P_{s}+P_{s}\left(\widetilde{A}_{i s}^{j}\right)^{T}+Q_{i s}^{j}, \\
X_{i f}^{j}=\widetilde{A}_{i 22}^{j} P_{f}+P_{f}\left(\widetilde{A}_{i 22}^{j}\right)^{T}+Q_{i f}^{j}, \\
M=\widetilde{A}_{i 22}^{j} \Theta P_{s} \Theta^{T}-\widetilde{A}_{i 21}^{j} P_{s} \Theta^{T}+\left(\widetilde{A}_{i 22}^{j} \Theta P_{s} \Theta^{T}-\widetilde{A}_{i 21}^{j} P_{s} \Theta^{T}\right)^{T}, \\
\Theta=\sum_{k=1}^{N} \sum_{l=1}^{r_{k}}\left(\widetilde{A}_{l 22}^{k}\right)^{-1} \widetilde{A}_{l 21}^{k}, \\
Y_{i}^{j}=-\left.\sum_{k=1}^{N} \sum_{l=1}^{r_{k}}\left(\widetilde{A}_{l 22}^{k}\right)^{-1} \widetilde{A}_{l 21}^{k} P_{s}\right|_{(k, l) \neq(j, i)} .
\end{gathered}
$$

Proof. Let

$$
\begin{gathered}
P_{1}(\varepsilon)=\varepsilon P_{s}, \\
P_{2}(\varepsilon)=\varepsilon P_{2}=-\varepsilon \sum_{k=1}^{N} \sum_{l=1}^{r_{k}}\left(\widetilde{A}_{l 22}^{k}\right)^{-1} \widetilde{A}_{l 21}^{k} P_{s}, \\
P_{3}(\varepsilon)=P_{f}+\varepsilon P_{2} P_{s}^{-1} P_{2}^{T}, \\
Q_{i 1}^{j}(\varepsilon)=\varepsilon\left(Q_{i s}^{j}-\widetilde{A}_{i 12}^{j} Y_{i}^{j}-\left(\widetilde{A}_{i 12}^{j} Y_{i}^{j}\right)^{T}\right), \\
Q_{i 2}^{j}(\varepsilon)=-\left(\widetilde{A}_{i 22}^{j} Y_{i}^{j}+P_{f}\left(A_{i 12}^{j}\right)^{T}\right), \\
Q_{i 3}^{j}(\varepsilon)=\varepsilon^{-1} Q_{i f}^{j} .
\end{gathered}
$$

It follows from (40) that

$$
\left[\begin{array}{cc}
\varepsilon\left(Q_{i s}^{j}-\widetilde{A}_{i 12}^{j} Y_{i}^{j}-\left(\widetilde{A}_{i 12}^{j} Y_{i}^{j}\right)^{T}\right) & \star \\
-\left(\widetilde{A}_{i 22}^{j} Y_{i}^{j}+P_{f}\left(\widetilde{A}_{i 12}^{j}\right)^{T}\right) & \varepsilon^{-1} Q_{i f}^{j}
\end{array}\right]>0, \quad \forall \varepsilon>0
$$

that is,

$$
\left[\begin{array}{cc}
Q_{i 1}^{j}(\varepsilon) & \star \\
Q_{i 2}^{j}(\varepsilon) & Q_{i 3}^{j}(\varepsilon)
\end{array}\right]>0, \quad \forall \varepsilon>0 .
$$

We can rewrite (37a) and (37b) as

$$
\dot{\chi}(t)=\sum_{j=1}^{N} \sum_{i=1}^{r^{j}} \theta_{j} h_{i}^{j}\left[\left(\widetilde{A}_{i}^{j}(\varepsilon)\right) \chi(t)\right],
$$

where

$$
\chi=\left[\begin{array}{l}
\eta \\
z
\end{array}\right], \quad \widetilde{A}_{i}^{j}(\varepsilon)=\left[\begin{array}{cc}
\widetilde{A}_{i 11}^{j} & \widetilde{A}_{i 12}^{j} \\
\varepsilon^{-1} \widetilde{A}_{i 21}^{j} & \varepsilon^{-1} \widetilde{A}_{i 22}^{j}
\end{array}\right] .
$$

Let

$$
P(\varepsilon)=\left[\begin{array}{cc}
P_{1}(\varepsilon) & \star \\
P_{2}(\varepsilon) & P_{3}(\varepsilon)
\end{array}\right] .
$$

Define $X_{i 1}^{j}(\varepsilon), X_{i 2}^{j}(\varepsilon), X_{i 3}^{j}(\varepsilon)$ as

$$
\begin{aligned}
X_{i 1}^{j}(\varepsilon)= & \widetilde{A}_{i 11}^{j} P_{1}(\varepsilon)+\left(\widetilde{A}_{i 11}^{j} P_{1}(\varepsilon)\right)^{T} \\
& +\widetilde{A}_{i 12}^{j} P_{2}(\varepsilon)+\left(\widetilde{A}_{i 12}^{j} P_{2}(\varepsilon)\right)^{T}+Q_{i 1}^{j}(\varepsilon), \\
X_{i 2}^{j}(\varepsilon)= & \varepsilon^{-1} \widetilde{A}_{i 22}^{j} P_{2}(\varepsilon)+\varepsilon^{-1} \widetilde{A}_{i 21}^{j} P_{1}(\varepsilon) \\
& +P_{3}(\varepsilon)\left(\widetilde{A}_{i 12}^{j}\right)^{T}+\left(\widetilde{A}_{i 11}^{j} P_{2}^{T}(\varepsilon)\right)^{T}+Q_{i 2}^{j}(\varepsilon), \\
X_{i 3}^{j}(\varepsilon)= & \varepsilon^{-1}\left(\widetilde{A}_{i 22}^{j} P_{3}(\varepsilon)+\left(\widetilde{A}_{i 22}^{j} P_{3}(\varepsilon)\right)^{T}\right. \\
& \left.\quad+\widetilde{A}_{i 21}^{j} P_{2}^{T}(\varepsilon)+\left(\widetilde{A}_{i 21}^{j} P_{2}^{T}(\varepsilon)\right)^{T}+Q_{i 3}^{j}(\varepsilon)\right)
\end{aligned}
$$

Then, the closed-loop system is stable for $\forall \varepsilon \in\left(0, \varepsilon_{\max }\right]$. 
which are equivalent to

$$
\begin{gathered}
X_{i 1}^{j}(\varepsilon)=\varepsilon\left(\widetilde{A}_{i s}^{j} P_{s}+P_{s}\left(\widetilde{A}_{i s}^{j}\right)^{T}+Q_{i s}^{j}\right)=\varepsilon X_{i s}^{j}, \\
X_{i 2}^{j}(\varepsilon)=\varepsilon\left(P_{2} P_{s}^{-1} P_{2}^{T}\left(\widetilde{A}_{i 12}^{j}\right)^{T}+\left(\widetilde{A}_{i 11}^{j} P_{2}^{T}\right)^{T}\right)=\varepsilon X_{i 2}^{j}, \\
X_{i 3}^{j}(\varepsilon)=\varepsilon^{-1}\left(\widetilde{A}_{i 22}^{j} P_{f}+P_{f}\left(\widetilde{A}_{i 22}^{j}\right)^{T}+Q_{i f}^{j}+\varepsilon M\right) \\
=\varepsilon^{-1}\left(X_{i f}^{j}+\varepsilon M\right) .
\end{gathered}
$$

From LMIs (39), it follows that

$$
\left[\begin{array}{cc}
X_{i s}^{j} & \star \\
\varepsilon X_{i 2}^{j} & X_{i f}^{j}+\varepsilon M
\end{array}\right]<0, \quad \forall \varepsilon \in\left(0, \varepsilon_{\max }\right]
$$

which is equivalent to

$$
\left[\begin{array}{cc}
\varepsilon X_{i s}^{j} & \star \\
\varepsilon^{2} X_{i 2}^{j} & \varepsilon X_{i f}^{j}+\varepsilon^{2} M
\end{array}\right]<0, \quad \forall \varepsilon \in\left(0, \varepsilon_{\max }\right]
$$

which implies that

$$
\left[\begin{array}{cc}
\varepsilon X_{i s}^{j} & \star \\
\varepsilon X_{i 2}^{j} & \varepsilon^{-1} X_{i f}^{j}+M
\end{array}\right]<0, \quad \forall \varepsilon \in\left(0, \varepsilon_{\max }\right] .
$$

Since

$$
\begin{aligned}
& {\left[\begin{array}{cc}
\varepsilon X_{i s}^{j} & \star \\
\varepsilon X_{i 2}^{j} & \varepsilon^{-1} X_{i f}^{j}+M
\end{array}\right]} \\
& =\left[\begin{array}{cc}
X_{i 1}^{j}(\varepsilon) & \star \\
X_{i 2}^{j}(\varepsilon) & X_{i 3}^{j}(\varepsilon)
\end{array}\right] \\
& =\widetilde{A}_{i}^{j}(\varepsilon) P(\varepsilon)+P(\varepsilon)\left(\widetilde{A}_{i}^{j}(\varepsilon)\right)^{T}+Q_{i}^{j}(\varepsilon),
\end{aligned}
$$

it follows from (44) and (52) that

$$
\widetilde{A}_{i}^{j}(\varepsilon) P(\varepsilon)+P(\varepsilon)\left(\widetilde{A}_{i}^{j}(\varepsilon)\right)^{T}<0, \quad \forall \varepsilon \in\left(0, \varepsilon_{\max }\right]
$$

then

$$
\prod_{i}^{j} \triangleq P^{-1}(\varepsilon) \widetilde{A}_{i}^{j}(\varepsilon)+\left(\widetilde{A}_{i}^{j}(\varepsilon)\right)^{T} P^{-1}(\varepsilon)<0, \quad \forall \varepsilon \in\left(0, \varepsilon_{\max }\right]
$$

Define Lyapunov function

$$
V(\chi(t))=\chi^{T}(t) P^{-1}(\varepsilon) \chi(t)
$$

Computing the derivative of $V(\chi(t))$ along the trajectories of system (45) and taking into account (55), we have

$$
\begin{aligned}
\dot{V} & (\chi(t)) \\
& =2 \chi^{T}(t) P^{-1}(\varepsilon) \dot{\chi}(t) \\
& =2 \chi^{T}(t) P^{-1}(\varepsilon)\left(\sum_{j=1}^{N} \sum_{i=1}^{r^{j}} \theta_{j} h_{i}^{j}\left[\left(\widetilde{A}_{i}^{j}(\varepsilon)\right) \chi(t)\right]\right) \\
& =2 \sum_{j=1}^{N} \sum_{i=1}^{r^{j}} \theta_{j} h_{i}^{j} \chi^{T}(t) P^{-1}(\varepsilon)\left[\left(\widetilde{A}_{i}^{j}(\varepsilon)\right) \chi(t)\right] \\
& =\sum_{j=1}^{N} \sum_{i=1}^{r^{j}} \theta_{j} h_{i}^{j} \chi^{T}(t) \prod_{i}^{j}(\varepsilon) \chi(t)<0, \quad \forall \varepsilon \in\left(0, \varepsilon_{\max }\right] .
\end{aligned}
$$

Thus, the closed-loop system is stable for $\forall \varepsilon \in\left(0, \varepsilon_{\max }\right]$. This completes the proof.

Remark 8 . Stability bound problem of SPSS is challenging. The existing method in [28] is limited to linear SPSS. Theorem 7 describes an efficient method to compute stability bound of nonlinear SPSS. The best estimation of the stability bound can be obtained by the following optimization problem:

$$
\begin{array}{ll}
\underset{P_{s}>0, P_{f}>0, Q_{i s}^{j}>0, Q_{i f}^{j}>0}{\max } & \mathcal{E}_{\max } \\
\text { s.t. } & \text { LMIs (39) and (40), }
\end{array}
$$

which can be effectively solved by a one-dimensional search algorithm with the aid of LMI Control Toolbox in Matlab 7.0.

Remark 9. It is known that LMI-based stability conditions for T-S fuzzy systems are usually sufficient conditions [30]. Theorem 2 is derived by using common Lyapunov function, which may lead to conservatism in some cases. Our future work will try to reduce the conservatism by using piecewise quadratic Lyapunov functions and fuzzy Lyapunov functions, which have been demonstrated to be less conservative under certain assumptions [31].

\section{A Numerical Example}

To illustrate the proposed results, we consider the following singularly perturbed switched system composed of two modes.

Mode 1:

$$
\begin{aligned}
& \dot{x}(t)=-\sin (x(t))+z(t)+\arctan (u(t))+0.55 u(t) \\
& \varepsilon \dot{z}(t)=x(t)-z(t)+\arctan (u(t))+0.55 u(t) .
\end{aligned}
$$

Mode 2:

$$
\begin{aligned}
& \dot{x}(t)=-x(t)+z(t)+\arctan (u(t))+0.55 u(t), \\
& \varepsilon \dot{z}(t)=x(t)-z(t)+\arctan (u(t))+0.55 u(t) .
\end{aligned}
$$


For the individual systems, we choose the interpolation points set as follows.

Mode 1:

$$
\begin{aligned}
O_{1}=\{ & (0,0,0),(0,0, \pm 2),(0,0, \pm 4), \\
& \left( \pm 60^{\circ}, 0,0\right),\left( \pm 60^{\circ}, 0, \pm 2\right),\left( \pm 60^{\circ}, 0, \pm 4\right), \\
& \left.\left( \pm 90^{\circ}, 0,0\right),\left( \pm 90^{\circ}, 0, \pm 2\right),\left( \pm 90^{\circ}, 0, \pm 4\right)\right\} .
\end{aligned}
$$

Mode 2:

$$
\mathrm{O}_{2}=\{(0,0,0),(0,0, \pm 2),(0,0, \pm 4)\} \text {. }
$$

Using Algorithm 2.1 in [42], we have the T-S fuzzy models of the system.

Mode 1:

$$
\begin{aligned}
& A_{111}^{1}=-1, \quad A_{112}^{1}=1, \quad A_{121}^{1}=1, \\
& A_{122}^{1}=-1, \quad B_{11}^{1}=1.55, \quad B_{12}^{1}=1.55, \\
& A_{211}^{1}=-1, \quad A_{212}^{1}=1, \quad A_{221}^{1}=1, \\
& A_{222}^{1}=-1, \quad B_{21}^{1}=1.1036, \quad B_{22}^{1}=1.1036, \\
& A_{311}^{1}=-1, \quad A_{312}^{1}=1, \quad A_{321}^{1}=1, \\
& A_{322}^{1}=-1, \quad B_{31}^{1}=0.8815, \quad B_{32}^{1}=0.8815 \text {, } \\
& A_{411}^{1}=-0.6366, \quad A_{412}^{1}=1, \quad A_{421}^{1}=1, \\
& A_{422}^{1}=-1, \quad B_{41}^{1}=1.55, \quad B_{42}^{1}=1.55, \\
& A_{511}^{1}=-0.6366, \quad A_{512}^{1}=1, \quad A_{521}^{1}=1, \\
& A_{522}^{1}=-1, \quad B_{51}^{1}=1.1036, \quad B_{52}^{1}=1.1036, \\
& A_{611}^{1}=-0.6366, \quad A_{612}^{1}=1, \quad A_{621}^{1}=1, \\
& A_{622}^{1}=-1, \quad B_{61}^{1}=0.8815, \quad B_{62}^{1}=0.8815 \text {, } \\
& A_{711}^{1}=0, \quad A_{712}^{1}=1, \quad A_{721}^{1}=1, \\
& A_{722}^{1}=-1, \quad B_{71}^{1}=1.55, \quad B_{72}^{1}=1.55, \\
& A_{811}^{1}=0, \quad A_{812}^{1}=1, \quad A_{821}^{1}=1, \\
& A_{822}^{1}=-1, \quad B_{81}^{1}=1.1036, \quad B_{82}^{1}=1.1036, \\
& A_{911}^{1}=0, \quad A_{912}^{1}=1, \quad A_{921}^{1}=1, \\
& A_{922}^{1}=-1, \quad B_{91}^{1}=0.8815, \quad B_{92}^{1}=0.8815 \text {. }
\end{aligned}
$$
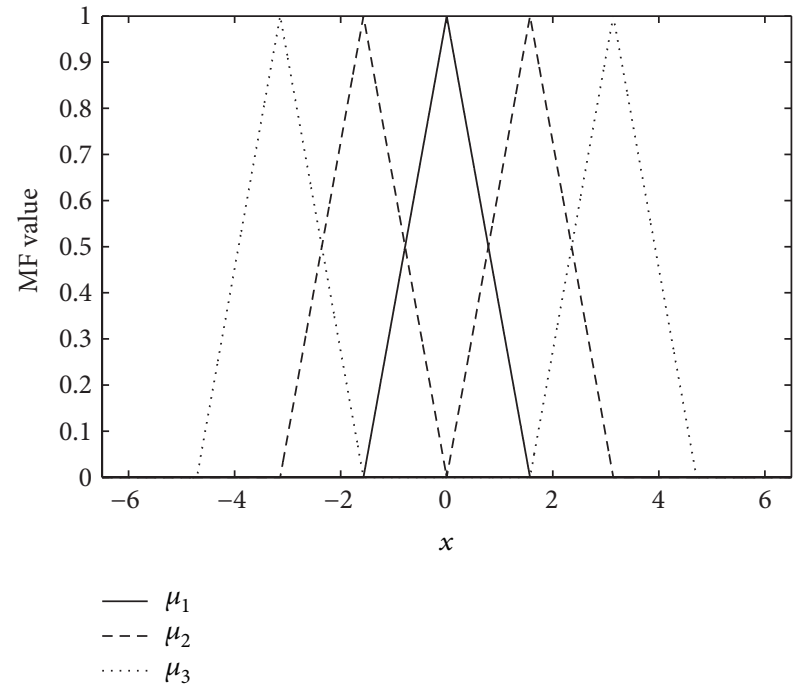

Figure 1: Membership function of $x$.

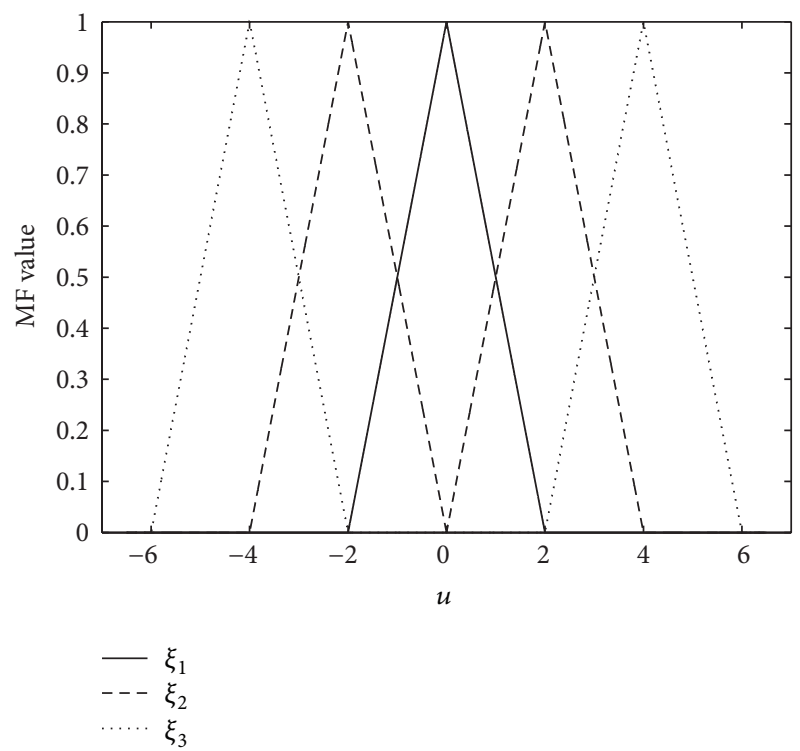

Figure 2: Membership function of $u$.

Mode 2:

$$
\begin{array}{ccl}
A_{111}^{2}=-1, & A_{112}^{2}=1, & A_{121}^{2}=1, \\
A_{122}^{2}=-1, & B_{11}^{2}=1.55, & B_{12}^{2}=1.55, \\
A_{211}^{2}=-1, & A_{212}^{2}=1, & A_{221}^{2}=1, \\
A_{222}^{2}=-1, \quad B_{21}^{2}=1.1036, & B_{22}^{2}=1.1036, \\
A_{311}^{2}=-1, \quad A_{312}^{2}=1, & A_{321}^{2}=1, \\
A_{322}^{2}=-1, \quad B_{31}^{2}=0.8815, & B_{32}^{2}=0.8815 .
\end{array}
$$

Triangle-type membership functions are employed as the fuzzy basis functions (see Figures 1 and 2 for the details). 


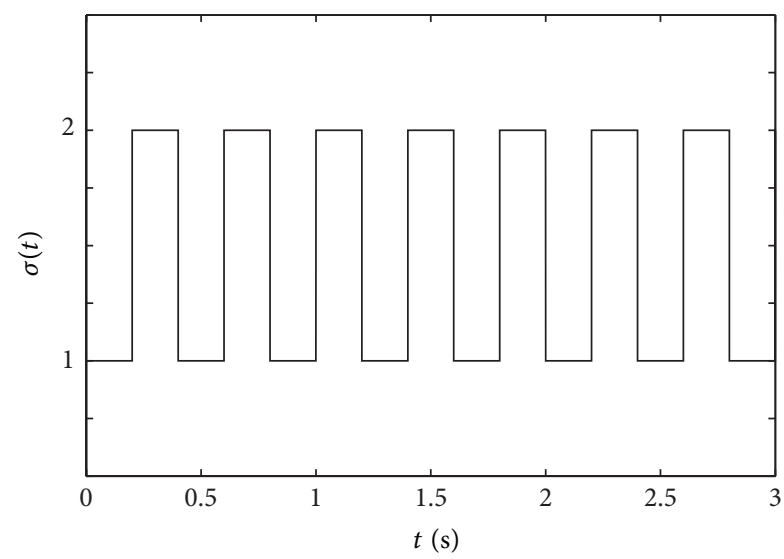

Figure 3: Switching rule $\sigma(t)$

Solving the LMIs in Theorem 2, we have the stabilizing controller gains:

$$
\begin{aligned}
& \bar{K}_{1}^{1}=\left[\begin{array}{lll}
-291.3404 & -246.0669 & 0.0486
\end{array}\right], \\
& \bar{K}_{2}^{1}=\left[\begin{array}{lll}
-254.0467 & -214.4164 & -0.0100
\end{array}\right], \\
& \bar{K}_{3}^{1}=\left[\begin{array}{lll}
-230.2670 & -194.1520 & -0.0237
\end{array}\right], \\
& \bar{K}_{4}^{1}=\left[\begin{array}{lll}
-267.1146 & -225.4624 & 0.0519
\end{array}\right], \\
& \bar{K}_{5}^{1}=\left[\begin{array}{lll}
-230.0488 & -194.0072 & -0.0113
\end{array}\right], \\
& \bar{K}_{6}^{1}=\left[\begin{array}{lll}
-205.9095 & -173.4338 & -0.0298
\end{array}\right], \\
& \bar{K}_{7}^{1}=\left[\begin{array}{lll}
-224.7013 & -189.3868 & -0.0639
\end{array}\right], \\
& \bar{K}_{8}^{1}=\left[\begin{array}{lll}
-188.2509 & -158.4596 & -0.0166
\end{array}\right], \\
& \bar{K}_{9}^{1}=\left[\begin{array}{lll}
-164.7476 & -138.4243 & -0.0524
\end{array}\right], \\
& \bar{K}_{1}^{2}=\left[\begin{array}{lll}
-291.3404 & -246.0669 & -0.0486
\end{array}\right], \\
& \bar{K}_{2}^{2}=\left[\begin{array}{lll}
-254.0467 & -214.4164 & -0.0100
\end{array}\right], \\
& \bar{K}_{3}^{2}=\left[\begin{array}{lll}
-230.2670 & -194.1520 & -0.0237
\end{array}\right],
\end{aligned}
$$

Under this controller, the stability bound of the closedloop system is $\varepsilon_{\max }=0.0685$ by using Theorem 7 .

For simulation, we choose $\varepsilon=0.01, x(0)=1, z(0)=$ $-1, u(0)=0$, and the switching rule shown in Figure 3. Applying the fuzzy controller to the original system, the state trajectories and control input of the closed-loop system are shown in Figure 4. It can be seen that the closed-loop system is stable.

\section{Conclusion}

In this paper, we considered the problem of fuzzy controller design for nonaffine-in-control singularly perturbed

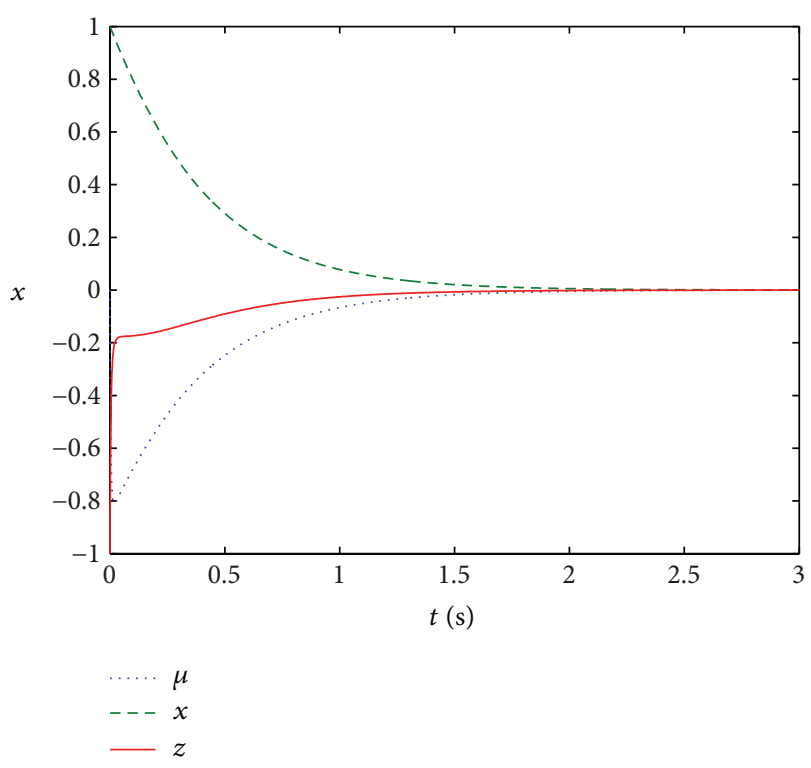

FIgURE 4: State trajectories and control input.

switched systems (NCSPSSs). A general class of TakagiSugeno (T-S) models including both state and control variables in the premise part of the rules were established to approximate the NCSPSS. By LMI technique, the controller design and stability bound estimation problems were solved. The presented example demonstrated the feasibility and effectiveness of the obtained methods. It can be seen that the proposed results are limited to standard NCSPSS. Thus, one of our future works is to investigate control and analysis problems of nonstandard NCSPSS.

\section{Conflict of Interests}

The authors declare that there is no conflict of interests regarding the publication of this paper.

\section{Acknowledgments}

This work was supported by the National Natural Science Foundation of China (61374043, 60904009, and 61020106003), the Jiangsu Provincial Natural Science Foundation of China (BK20130205), the China Postdoctoral Science Foundation funded Project (2013M530278, 2014T70558), the Fundamental Research Funds for the Central Universities (2013QNA50, 2013RC10, 2013RC12, and 2013XK09), and the Natural Science Foundation of Liaoning Province (201202201).

\section{References}

[1] D. Liberzon and A. S. Morse, "Basic problems in stability and design of switched systems," IEEE Control Systems Magazine, vol. 19, no. 5, pp. 59-70, 1999.

[2] D. Liberzon, Switching in Systems and Control, Birkhäuser, Boston, Mass, USA, 2003. 
[3] J. Zhao and G. M. Dimirovski, "Quadratic stability of a class of switched nonlinear systems," IEEE Transactions on Automatic Control, vol. 49, no. 4, pp. 574-578, 2004.

[4] H. Lin and P. J. Antsaklis, "Stability and stabilizability of switched linear systems: a survey of recent results," IEEE Transactions on Automatic Control, vol. 54, no. 2, pp. 308-322, 2009.

[5] A. Hocine, M. Chadli, and H. R.. Karimi, "A structured filter for Markovian switching systems," International Journal of Systems Science, vol. 45, no. 7, pp. 1518-1527, 2014.

[6] M. Darouach and M. Chadli, "Admissibility and control of switched discrete-time singular systems," Systems Science \& Control Engineering, vol. 1, no. 1, pp. 43-51, 2013.

[7] M. Chadli and M. Darouach, "Robust admissibility of uncertain switched singular systems," International Journal of Control, vol. 84, no. 10, pp. 1587-1600, 2011.

[8] H. Ishii and B. A. Francis, "Stabilizing a linear system by switching control with dwell time," IEEE Transactions on Automatic Control, vol. 47, no. 12, pp. 1962-1973, 2002.

[9] P. V. Kokotovic, H. K. Khalil, and J. O’Reilly, Singularly Perturbation Methods in Control: Analysis and Design, Academic Press, New York, NY, USA, 1986.

[10] D. S. Naidu, "Singular perturbations and time scales in control theory and applications: an overview," Dynamics of Continuous, Discrete and Impulsive Systems Series B: Applications and Algorithms, vol. 9, no. 2, pp. 233-278, 2002.

[11] I. A. Gondal, "On the application of singular perturbation techniques to nuclear engineering control problems," IEEE Transactions on Nuclear Science, vol. 35, no. 5, pp. 1080-1085, 1988.

[12] J.-W. Son and J.-T. Lim, "Stabilization of approximately feedback linearizable systems using singular perturbation," IEEE Transactions on Automatic Control, vol. 53, no. 6, pp. 1499-1503, 2008.

[13] A. Zagaris, H. G. Kaper, and T. J. Kaper, "Analysis of the computational singular perturbation reduction method for chemical kinetics," Journal of Nonlinear Science, vol. 14, no. 1, pp. 59-91, 2004.

[14] J. W. Kimball and P. T. Krein, "Singular perturbation theory for DC-DC converters and application to PFC converters," IEEE Transactions on Power Electronics, vol. 23, no. 6, pp. 2970-2981, 2008.

[15] A. E. Lindsay, J. Lega, and F. J. Sayas, "The quenching set of a MEMS capacitor in two-dimensional geometries," Journal of Nonlinear Science, vol. 23, no. 5, pp. 807-834, 2013.

[16] H. D. Tuan and S. Hosoe, "Multivariable circle criteria for multiparameter singularly perturbed systems," IEEE Transactions on Automatic Control, vol. 45, no. 4, pp. 720-725, 2000.

[17] H. Liu, F. Sun, and Y. Hu, " $H_{\infty}$ control for fuzzy singularly perturbed systems," Fuzzy Sets and Systems, vol. 155, no. 2, pp. 272-291, 2005.

[18] L. Saydy, "New stability/performance results for singularly perturbed systems," Automatica, vol. 32, no. 6, pp. 807-818, 1996.

[19] L. Cao and H. M. Schwartz, "Complementary results on the stability bounds of singularly perturbed systems," IEEE Transactions on Automatic Control, vol. 49, no. 11, pp. 2017-2021, 2004.

[20] I. Malloci, J. Daafouz, and C. Tung, "Stability and stabilization of two time scale switched systems in discrete time," IEEE Transactions on Automatic Control, vol. 55, no. 6, pp. 1434-1438, 2010.

[21] I. Malloci, J. Daafouz, and C. Iung, "Stabilization of continuoustime singularly perturbed switched systems," in Proceedings of the Joint 48th IEEE Conference on Decision and Control Held Jointly with the 28th Chinese Control Conference (CDC/CCC '09), pp. 6371-6376, Shanghai, China, December 2009.

[22] I. Malloci, J. Daafouz, C. Iung, R. Bonidal, and P. Szczepanski, "Switched system modeling and robust steering control of the tail end phase in a hot strip mill," Nonlinear Analysis: Hybrid Systems, vol. 3, no. 3, pp. 239-250, 2009.

[23] E. H. Abed, "A new parameter estimate in singular perturbations," Systems \& Control Letters, vol. 6, no. 3, pp. 193-198, 1985.

[24] H. K. Khalil, "Stability analysis of nonlinear multiparameter singularly perturbed systems," IEEE Transactions on Automatic Control, vol. 32, no. 3, pp. 260-263, 1987.

[25] M. S. Alwan, X. Liu, and B. Ingalls, "Exponential stability of singularly perturbed switched systems with time delay," Nonlinear Analysis: Hybrid Systems, vol. 2, no. 3, pp. 913-921, 2008.

[26] M. S. Alwan and X. Liu, "Stability of singularly perturbed switched systems with time delay and impulsive effects," Nonlinear Analysis: Theory, Methods \& Applications, vol. 71, no. 9, pp. 4297-4308, 2009.

[27] I. Malloci, J. Daafouz, C. Iung, R. Bonidal, and P. Szczepanski, "Robust steering control of hot strip mill," IEEE Transactions on Control Systems Technology, vol. 18, no. 4, pp. 908-917, 2010.

[28] G. S. Deaecto, J. Daafouz, and J. C. Geromel, " $\mathrm{H}_{2}$ and $H_{\infty}$ performance optimization of singularly perturbed switched systems," SIAM Journal on Control and Optimization, vol. 50, no. 3, pp. 1597-1615, 2012.

[29] T. Takagi and M. Sugeno, "Fuzzy identification of systems and its applications to modeling and control," IEEE Transactions on Systems, Man and Cybernetics, vol. 15, no. 1, pp. 116-132, 1985.

[30] G. Feng, "A survey on analysis and design of model-based fuzzy control systems," IEEE Transactions on Fuzzy Systems, vol. 14, no. 5, pp. 676-697, 2006.

[31] G. Feng, Analysis and Synthesis of Fuzzy Control Systems: A Model Based Approach, CRC Press, Boca Raton, Fla, USA, 2010.

[32] Y. M. Chen, C. M. Lin, and C. S. Hsueh, "Emitter identification of electronic intelligence system using type-2 fuzzy classifier," Systems Science and Control Engineering, vol. 2, no. 1, pp. 389397, 2014.

[33] A. Ghanbari and M. R. Moghanni-Bavil-Olyaei, "Adaptive fuzzy terminal sliding-mode control of MEMS $z$-axis gyroscope with extended Kalman filter observer," Systems Science \& Control Engineering, vol. 2, no. 1, pp. 183-191, 2014.

[34] A. Iqbal, H. Abu-Rub, and H. Nounou, "Adaptive fuzzy logiccontrolled surface mount permanent magnet synchronous motor drive," Systems Science \& Control Engineering, vol. 2, no. 1, pp. 465-475, 2014.

[35] H. Liu, F. Sun, and Z. Sun, "Stability analysis and synthesis of fuzzy singularly perturbed systems," IEEE Transactions on Fuzzy Systems, vol. 13, no. 2, pp. 273-284, 2005.

[36] W. Assawinchaichote and S. K. Nguang, "Fuzzy $H_{\infty}$ output feedback control design for singularly perturbed systems with pole placement constraints: an LMI approach," IEEE Transactions on Fuzzy Systems, vol. 14, no. 3, pp. 361-371, 2006.

[37] G.-H. Yang and J. Dong, "Control synthesis of singularly perturbed fuzzy systems," IEEE Transactions on Fuzzy Systems, vol. 16, no. 3, pp. 615-629, 2008.

[38] C. Yang and Q. Zhang, "Multiobjective control for T-S fuzzy singularly perturbed systems," IEEE Transactions on Fuzzy Systems, vol. 17, no. 1, pp. 104-115, 2009. 
[39] H. M. Gutierrez and P. I. Ro, "Magnetic servo levitation by sliding-mode control of nonaffine systems with algebraic input invertibility," IEEE Transactions on Industrial Electronics, vol. 52, no. 5, pp. 1449-1455, 2005.

[40] A. Young, C. Y. Cao, N. Hovakimyan, and E. Lavretsky, "Control of a nonaffine doublependulum system via dynamic inversion and timescale separation," in Proceedings of the American Control Conference, pp. 1820-1825, Minneapolis, Minn, USA, June 2006.

[41] S. S. Ge, C. C. Hang, and T. Zhang, "Nonlinear adaptive control using neural networks and its application to CSTR systems," Journal of Process Control, vol. 9, no. 4, pp. 313-323, 1999.

[42] Q. Gao, G. Feng, Y. Wang, and J. Qiu, "Universal fuzzy controllers based on generalized T-S fuzzy models," Fuzzy Sets and Systems, vol. 201, pp. 55-70, 2012.

[43] Q. Gao, X. J. Zeng, G. Feng, Y. Wang, and J. Qiu, “T-S-fuzzymodel-based approximation and controller design for general nonlinear systems," IEEE Transactions on Systems, Man, and Cybernetics, Part B: Cybernetics, vol. 42, no. 4, pp. 1143-1154, 2012.

[44] S. Boyd, L. El Ghaoui, E. Feron, and V. Balakrishnan, Linear Matrix Inequalities in System and Control Theory, SIAM, Philadelphia, Pa, USA, 1994. 


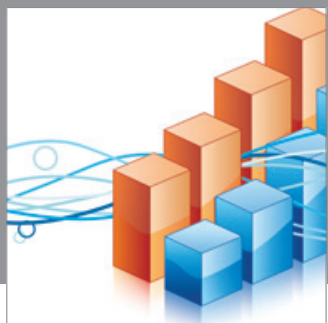

Advances in

Operations Research

mansans

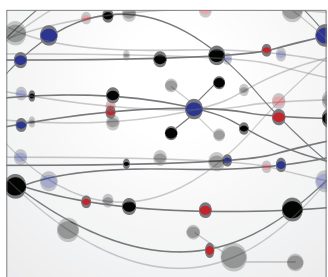

The Scientific World Journal
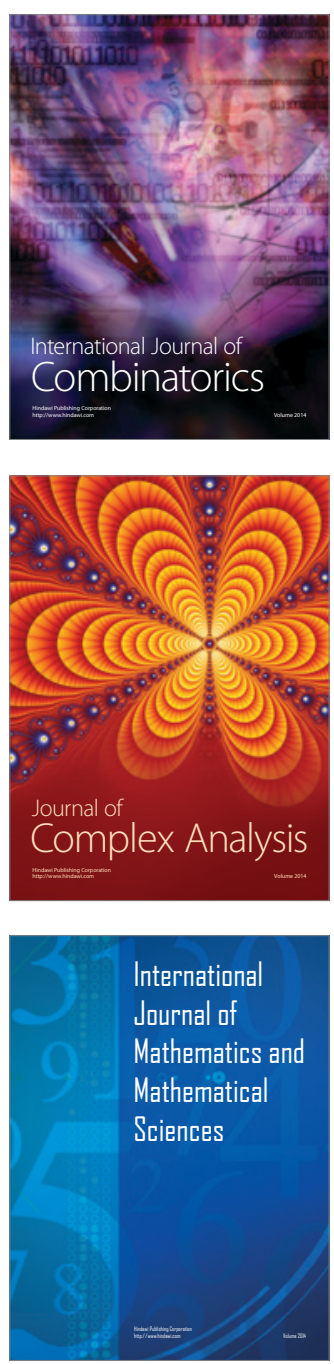
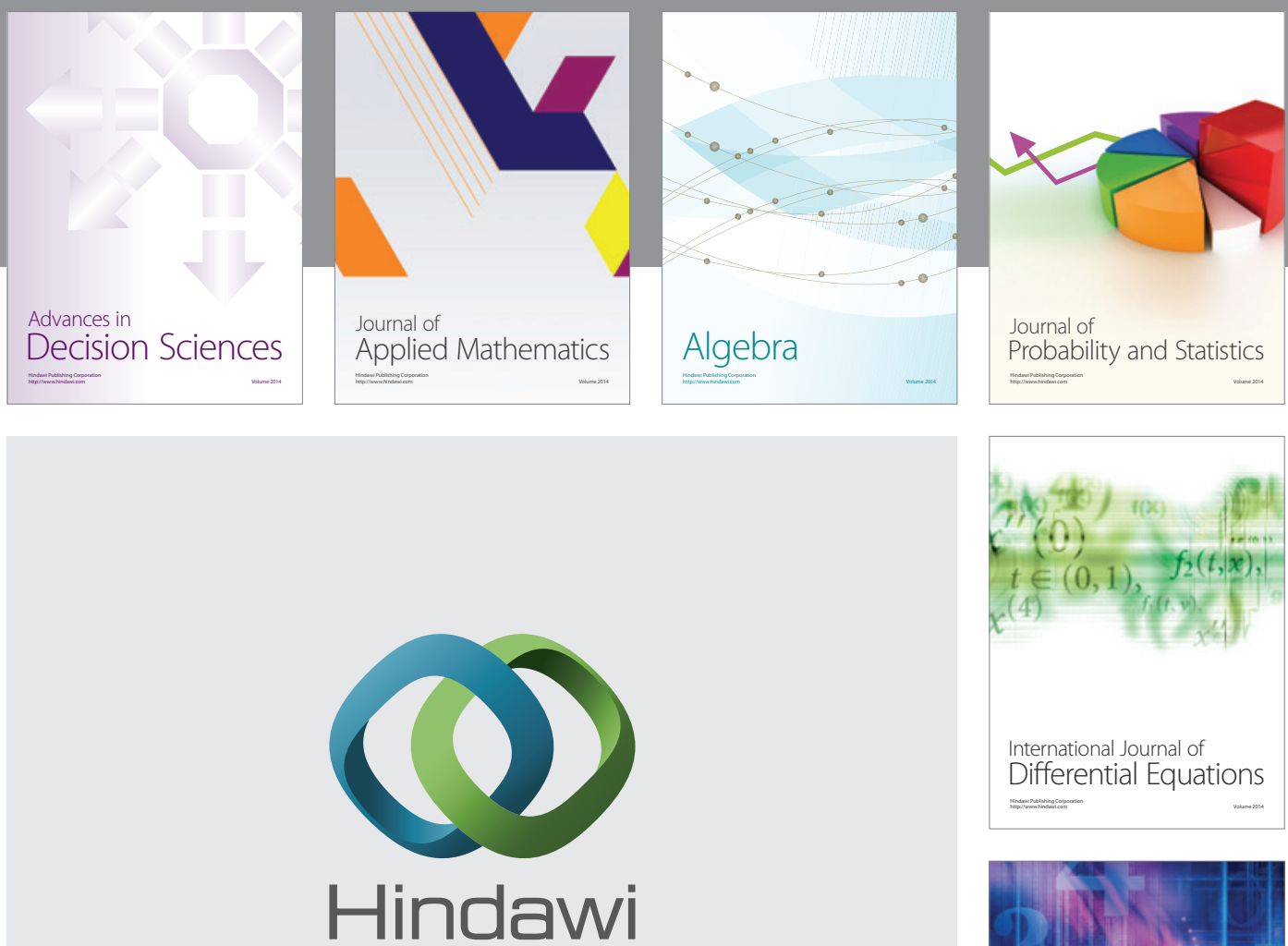

Submit your manuscripts at http://www.hindawi.com
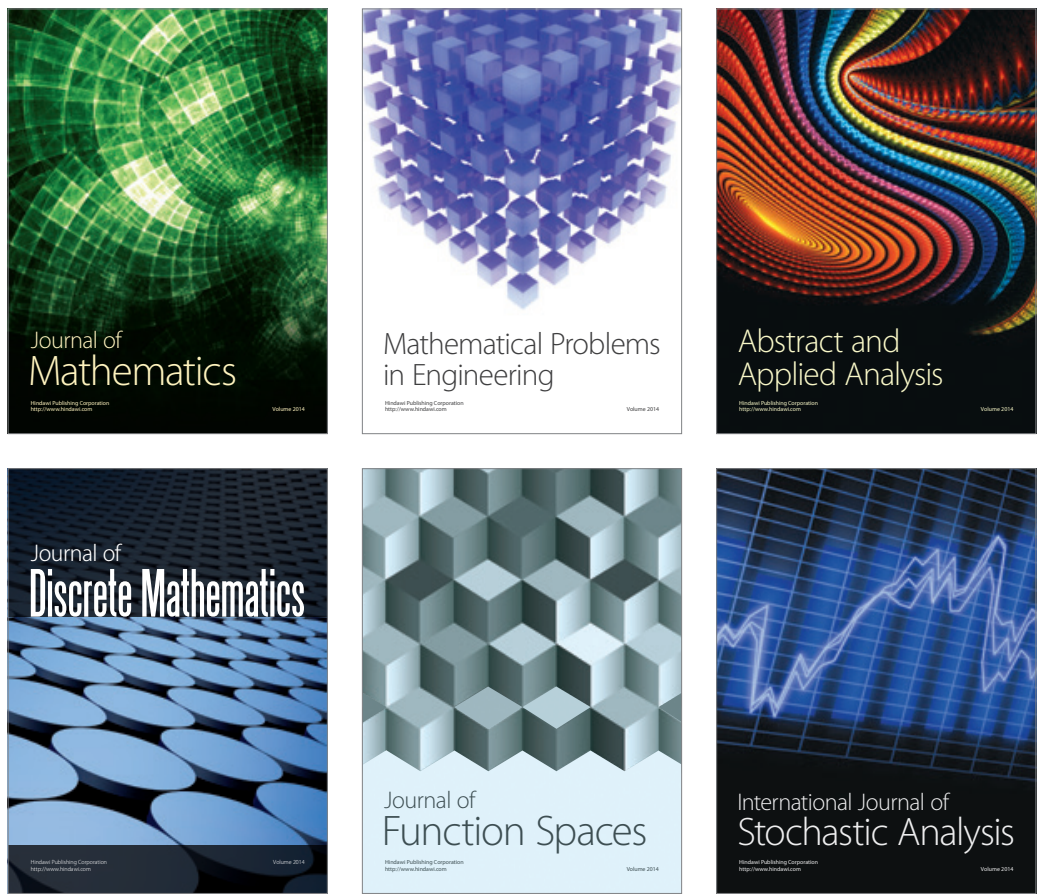

Journal of

Function Spaces

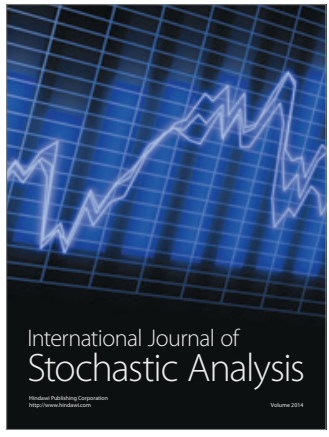

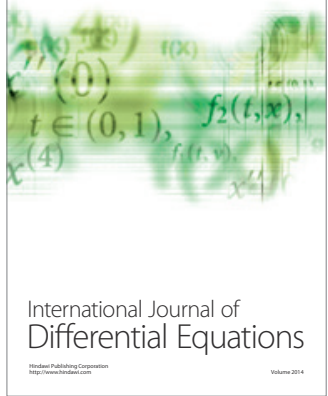
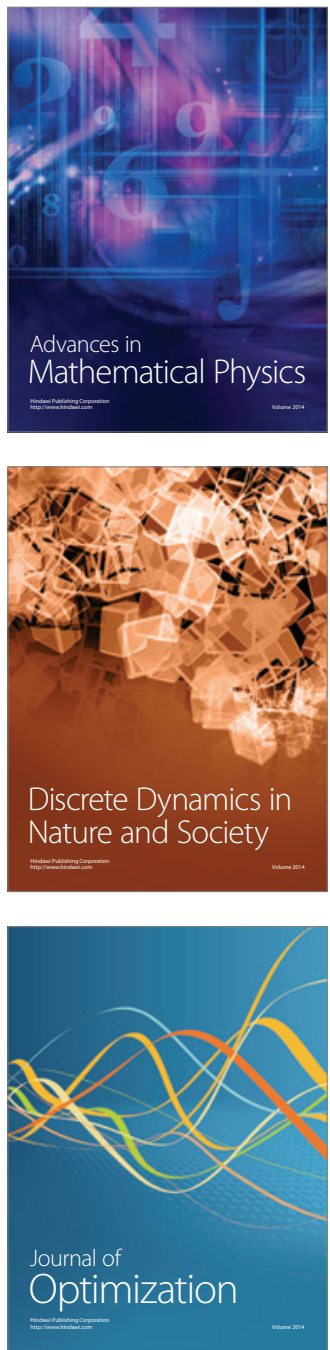